\title{
Individual Psychological Conflict within Social Group Dynamics
}

\author{
W.T. Johnson $^{\text {a }}$, V.G. Ivancevic ${ }^{\mathrm{a}}$ \\ ${ }^{\mathrm{a}} \mathrm{LD}$ and JOAD, Defence Science and Technology Organisation, Australia. \\ Email: wayne.johnson@dsto.defence.gov.au
}

\begin{abstract}
In this paper we investigate the implications of a conceptual-model for competition in decisionmaking, where as a result psychological conflict might arise for either individuals or social groups of artificial embodied agents. We propose a nonlinear dynamical system with control approach to compose a sociocognio-physical model of embodied agents in our crowd dynamics simulator, with a Lewin field theoretic model of cognition, which in principal is capable of being applied to a wide range of concrete problems. For instance, elsewhere in this conference Millikan et al. (2013) applies these Lewinian principles to the problem of military suppression for dismounted infantry. Wong (2012) has also used these constructs in a study on crowd reactions to Non-Lethal-Weapons.
\end{abstract}

Of Lewin's nine constructs of field-theory we will largely be concerned with the constructs of conflict (approach-avoid psychological conflict amongst the typology of Lewinian psychological conflicts) position, locomotion, force, goal and will briefly touch on power (inducing psychological forces on others to cause them to act; e.g. command). In particular we present a brief recount of psychological conflict simulation models, together with a qualitative analysis of conflicting attractor behaviour. Further, the consequences of the field shape have been considered for a simple nonlinear model of attraction and repulsion. There are a number of reasons why consideration of complex nonlinearity is required for modelling embodied cognition. Among them, we note, it is not possible to look at individual behaviours in complete isolation (classic controlled psychology experiments), without breaking the whole web of interdependencies (Lewin, 1997, pp 383-410).

Methods of investigation for general social dynamics have been reviewed by Castellano et al. (2009) as being entropic, topological, dynamical systems (DS) and agent based models (ABM). Castellano's statistical-physics based approach, however, fails to identify explicit individual entity control or self-regulation as an important element; see (Conant and Ashby, 1970). Therefore the minimum modelling capability of an embodied agent must be effectively the equivalent of a dynamical system with control (DSC), irrespective of whether the model implementation is actually an ABM or alternatively a nonlinear DS with each agent possessing a cybernetic controller for its cognitive model.

While there are other computational models of cognition ranging from the disembodied to the social, see (Sun, 2008) for summaries on SOAR, ACT-R, CLARION, they cannot cope with the change in scale of modelling fidelity, because, in our view, they do not see their psychological and physical worlds in terms of fundamental forces, be they physical, psychological or social, in some appropriate geometrical space. Neither can they seamlessly recurse between a representation of the psychological-present moment and either the psychological-past or a projected psychological-future, or indeed both; see (Ivancevic and Johnson, 2012). They also fail to represent an explicit model of control (Johnson et al., 2011), breaking Ashby's dictum that "Every good regulator of a system must be a model of that system" (Conant and Ashby, 1970).

In brief then, our broad problem is to find predictive embodied models of cognition with explicit models of control or self-regulation. We are following Millikan et al. (2011), who have suggested that understanding the detailed behaviour of conceptual models is a key part of a wider effort toward holistic societal modelling. In particular, the conceptual model under investigation here is one of nonlinear psychological competition in decision-making, where the competition may lead to psychological conflict and vacillatory behaviour or stable equilibria. The specific question we will examine in this study is the effect of the dynamic spatiotemporal field-strength shape on the behaviour of artificial embodied-agents. Following a standard approach used in theoretical-physics we will progressively examine field-strengths which vary linearly, then cubically and finally quintically. Further, appropriate control geometries and are being sought, following Lewin (1936), to represent the dynamics of individual agents and social-groups decision making interactions.

Keywords: Crowd dynamics, Lewin's field-theory, Psychological approach-avoid conflict 


\section{INTRODUCTION}

The most important requirement is that, in practice, in order to predict, we need to calculate. This, of course, implies a computational model of individually-based agent motivation; see (Johnson et al., 2011). It is taken for granted here that any agent-capability should sense, decide and act while situated in some world; see (Ferber, 1999). For low level ABMs the decide-function is the cognitive-model. Similarly for nonlinear DSCs the cybernetic-controller is the nonlinear self-regulator or in our case the nonlinear cognitive-model; see (Conant and Ashby, 1970). The interactions that we are interested in are between the embodied agent and firstly, the physical world, secondly, his individual psychological world and finally the in-groups and out-groups of his social world, and between these three broad groups it is trivially obvious that the system is fundamentally nonlinear. In particular we examine the effect of the dynamic spatio-temporal potential field-strength shape on the behaviour of artificial embodied-agents.

It is, of course, desirable to have a theoretical basis before proceeding. In our case it comes from Kurt Lewin's socio-cognio field-theory or life-space; see (Lewin, 1935, p.79). The traditional standard approach used in psychology is to examine only the linear case in terms of psychological-forces (Miller, 1944; Weiten, 1998), whereas we will progressively examine potential field-strengths which vary linearly, then cubically and finally quintically. This approach is more consistent with Lewin's field-theoretic view on the topological nature of the life-space (Lewin, 1936).

\subsection{Lewin's field-theory}

Lewin's most famous equation (first mentioned in (Lewin, 1935, pp.73,79)) can be formulated as a disjoint union of the following functional mappings (indicated by ' $\rightarrow$ ', for $i=1,2, \ldots ; a=1,2, \ldots ; \alpha=1,2, \ldots$ ):

$$
\bigcup_{i, a, \alpha}\left\{\left(P_{a}, E_{i}\right) \rightarrow B_{\alpha}\right\} \quad \Rightarrow \quad B=f(P, E) \quad \Leftrightarrow \quad B_{\alpha}=f\left(P_{a}, E_{i}\right)
$$

where $B=\left\{B_{\alpha}\right\}$ denotes the total behaviour composed from the set of the modes of behaviour $B_{\alpha} ; P=\left\{P_{a}\right\}$ is the person's total mental state consisting of the set of various mental states and structures $P_{a} ; E=\left\{E_{i}\right\}$ is the psycho-biological environment with differing environmental regions $E_{i}$. In toto, $f$ is a function of $P, E$, the essential components of the total situation. The first two terms in Lewin's equation are his own formulation, while the last term is a modern tensorial one. The environment he referred to was a psycho-biological-space and to distinguish it from the physical space, he called it life-space (Lewin, 1935, p.79).

Lewin was inspired by field-theoretic interpretations of classical and relativistic physics, with their Euclidean and Riemannian geometries, which he applied in his field-theory, comprising of what he called topological and vector psychology, (Lewin, 1936). The function $f$ in (1) is quite general and abstract, and we will see later how it possible to model the behaviours $B$ more specifically and concretely as either force-fields, $F$, as Lewin would have preferred, or as potential-fields, $V$, as we prefer ${ }^{1}$.

In brief, the nine constructs the field-theory of (Lewin, 1997, pp.197-198) were as follows:- 1. Position for location and the related geometry of relative position (i.e., topology); 2. Locomotion or relation of geometric position over time; 3. Cognitive structure for the relative position of various geometrical objects (points, boundaries, regions, etc.); 4. Force for the tendency to locomotion and change over time, and the accompanying force-field for the spatial distribution of forces; 5. Goal for a force-field focused on a particular point, with a positive valence; aversion is similar but with a negative valence; 6 . Conflict ${ }^{2}$ for at least two overlapping competing force-fields, which may result in a vacillatory equilibrium for certain constellations of overlapping force-fields; 7. Fear which is related to the psychological-future; 8. Power which refers to a "possibility of inducing forces" on another person via a power-field, e.g., a parent's power over a child; 9. Values refers to internal or self regulating power, which Lewin in comparison to Freud's psychoanalytical theory thought could be the internalized-parent (Lewin, 1997, pp.197-198). In this paper we will only be implicitly concerned with the constructs of position, locomotion, force, goal, and power, but our main focus will be on conflict, and the associated force-fields or potential fields.

\footnotetext{
${ }^{1}$ Potential functions will allow us to more easily add the contributions of other fields, e.g.. a gravitational field acting on the agent's body; see the crowd dynamics simulator in Section 2.

${ }^{2}$ This refers to psychological conflict within the life-space, not violence in a physical space.
} 


\subsection{Psychological conflict in decision-making}

For the present work we only need to consider the Lewinian typology of conflicts, (Lewin, 1935, p.88-91) and extensions, which are:- 1. approach-approach: we want two different things both of which we like (in Lewin's terms, both have 'positive valences'); 2. avoidance-avoidance: we have to pick one or the other alternative, but dislike both (both have 'negative valences'); 3. approach-avoidance: we both want and dislike differing aspects or qualities of the same thing (it has both positive and negative 'valences', but the gradient of avoid is assumed steeper than that of the approach, see (Miller, 1944, p.434)); 4. double-approach-avoidance: we must choose between two things that each have both positive and negative qualities (valences), see (Miller, 1944, p.446); 5. avoidance-approach: as for approach-avoid but explicitly with approach gradients greater than avoidance gradients; see (Epstein, 1978).

As an application of Lewinian force-field socio-cognio-physics in digital computer era, we can mention the simulations of Balkenius (1995); Jager et al. (2001, p.79-125) and Wong (2012) Jager et-al also simulated the approach-avoidance conflict in two-party crowds (Jager et al., 2001), but their simulation fails as a model of pure psychological approach-avoid conflict (Johnson et al., 2011), but can be considered a sociological model of approach and avoid; it also had no gradient of attraction. While Wong (2012) has only a nearest neighbour approximation to the approach-avoidance conflict Lewinian field in a riot control scenario, the simulation successfully produces a vacillatory equilibrium for individual agents in the crowd.

\section{THE PSYCHOLOGICAL CONFLICT MODEL}

Before we examine the psychological conflict model we need to introduce a little background on the entropictopological dynamics framework which accompanies the crowd dynamics simulator (CDS) (Ivancevic and Reid, 2010). Within the CDS it is important to understand that it represents entities flowing on a landscape of a heavily viscous fluid. Unless control-forces are acting upon the entities they effectively will be in Brownian motion. Thus far the space in which the CDS operates has been treated as physical space. There is no reason however, that it could not be purely a psychological life-space, effectively the pure cybernetic controller incorporating its own model of the physical space. The CDS as a systems-component can be reused to model both of these interpretations and their interconnection; see (Ivancevic and Johnson, 2012). Here we consider a pure life-space and examine the qualities of various psychological fields and the ensuing behaviours.

\subsection{A perturbation-theoretic qualitative analysis of the life-space field}

To the best of our knowledge, following (Miller, 1944; Weiten, 1998), psychologists have never considered the approach-avoidance behaviours in terms of nonlinear relationships. These considerations and the associated qualitative analysis form part of the novel contribution of the current work. Throughout the following we will examine, via a qualitative analysis, the consequences of modelling Lewin's general behavioural function $f$, in (1), as either a force-field, $F$, or as a potential-field, $V$.

Linear forces. Adding the linear strength-distance conflict relation into the crowd dynamics simply means adding a linear term to the general agent's velocity controller and modelling (1) as:

$$
B=f(P, E) \quad \Leftrightarrow \quad B=f(k, x) \equiv F(k, x)=-k x .
$$

in which the force-field is (negatively) proportional to the agent's perceived displacement $x$ from the source of attraction of attraction/repulsion; see the r.h.s. Note that $f \equiv F$, that is, we are modelling behaviours $B$ as force-fields, with $P=k$, the individual agent's mental state of strength of attraction to the source, represented by the constant parameter $k$, and $E=x$, as the representation of the one dimensional environmental region of the source centred at $x=0$. To incorporate the simplified strength-distance relation on the r.h.s. of (2) into the CDS, we firstly consider a simple Newtonian model $(F=m \ddot{x})$ of a damped linear oscillator of mass $m$ in a strongly dissipative environment with viscosity $\nu$. We have:

$$
m \ddot{x}+\nu \dot{x}+k x=0, \quad \text { or } \quad \ddot{x}+\xi \dot{x}+\omega_{0}^{2} x=0,
$$

where $\xi=\nu / m$, while $\omega_{0}=\sqrt{k / m}$ is the natural frequency of the damped oscillations of the linear oscillator (3). The assumed case of strongly-dissipative environment, $|\xi| \gg 1$, allows us to neglect the inertial term in (3), so we end up with a simple kinematic equation:

$$
\dot{x}=-\alpha x \equiv F(x), \quad \text { where } \quad \alpha=\omega_{0}^{2} / \xi .
$$



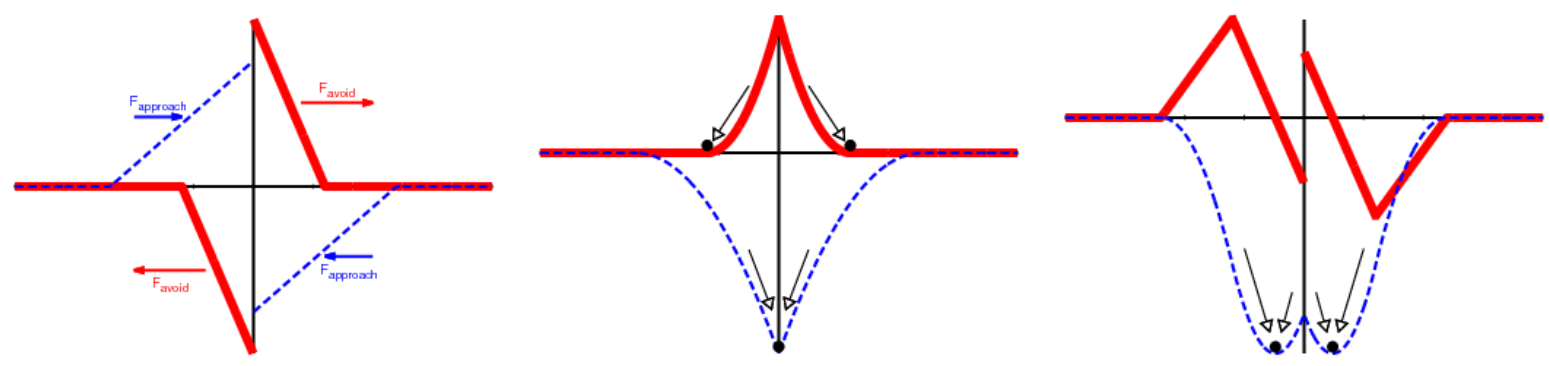

Figure 1. Classic psychological approach avoid vacillation behaviour. Left: The dashed line represents the linear psychological 'forces' of attraction while the solid line represents linear psychological 'forces' of repulsion. Middle: The dashed line represents the potential function of attraction $V_{\text {approach }}(x)$ while the solid line represents potential function of repulsion $V_{\text {avoid }}(x)$ Right: The dashed line represents the total or resultant potential well $V_{\text {total }}(x)$, while solid line represents the total or resultant linear 'forces' of attraction and repulsion. Note the vacillation point is offset from the origin.

Our simple dissipative kinematics (4) represents a particular form of the general velocity controller. It can be visualized as a motion of a particle in a potential well $V(x)$, such that $F(x)=-\frac{\partial V}{\partial x}$ :

$$
B=f(P, E) \quad \Leftrightarrow \quad B=V(\alpha, x)=\frac{1}{2} \alpha x^{2} .
$$

Here, we are now concretely modelling behaviours $B$ as a potential function; i.e. $f \equiv V$, and $\alpha$ is a parameter. From the simple quadratic shape of the potential well $V(x)$, it is clear that the system (4), in the case of positive 'frequency/damping ratio' $\alpha=\omega_{0}^{2} / \xi>0$, has just one stable stationary state: the minimum $x=0$ of the potential $V(x)$. However overlapping linear fields with differing gradients will produce the classic approach avoidance result (Weiten, 1998); see Figure 1.

To make it a better representation of the socio-cognio-physics of the real world, some nonlinear enhancements to the attractor and repeller formulation are required. Secondly, to make our conflicting strength-distance behaviour more realistic, we can allow the parameter $\alpha=\omega_{0}^{2} / \xi$ to become time-dependent, that is $\alpha=\alpha(t)$, so that we obtain the more general (but still linear), time-varying, kinematic system: $\dot{x}(t)=-\alpha(t) x(t)$, with the corresponding time-varying potential well: $V(\alpha, x, t)=\frac{1}{2} \alpha(t) x(t)^{2}$. This implies an explicit generalisation of (1) such that $B=f(P, E)$ now becomes $B=f(P, E, t)$.

Cubic forces. Now, let us consider a nonlinear modification of the linear conflicting strength-distance relation (2), modelled this time by nonlinearly. So, if we add a similar cubic nonlinearity to the linear and stable kinematic system (4), we obtain the following nonlinear and time-varying agent conflict model:

$$
\dot{x}(t)=-\alpha(t) x(t)-\beta(t) x(t)^{3} .
$$

Its corresponding evolving conflict potential well is given by the time-varying polynomial:

$$
B=f(P, E, t) \quad \Leftrightarrow \quad B=V(\alpha, \beta, x, t)=\frac{1}{2} \alpha(t) x(t)^{2}+\frac{1}{4} \beta(t) x(t)^{4} .
$$

In the conflict model (6)-(7) we need to analyse three different scenarios (all with positive nonlinear parameter $\beta(t)>0)$ :

1. The case of positive 'frequency/damping ratio': $\alpha \geq 0$. In this case, the shape of potential well $V$ resembles the previous quadratic form with a single stable stationary state: the minimum $(x=0)$ of the potential well $V$. This can be justified by the fact that the cubic equation: $\alpha x+\beta x^{3}=0$ (with both positive parameters) has only one real root: $x=0$.

2. The case of negative 'frequency/damping ratio': $\alpha<0$. In this case, the the potential well $V$ has a symmetrical quartic shape with two minima (symmetrically positioned left and right from the origin), which are both stable stationary states. In this case the cubic equation: $\alpha x+\beta x^{3}=0$ has two real roots, $x_{1}<0$ and $x_{2}>0$. 


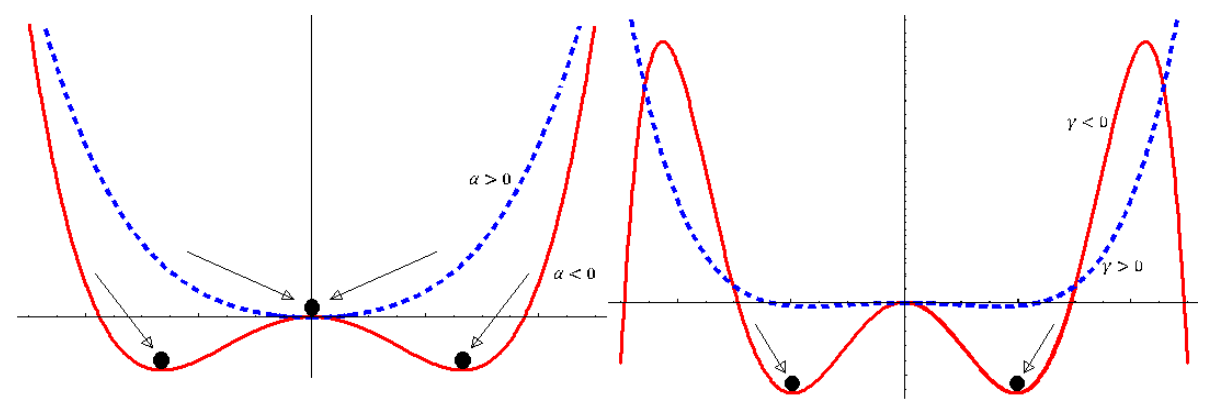

Figure 2. Dynamic nonlinear field behaviours. Left: Individual agent's extended, cubic, conflict potential well $V(\alpha, \beta, x, t)$ with bifurcating phase-transition from stability (dashed line) to bistability (solid line). Right: Individual agent's extended, quintic, conflict potential well $V(\alpha, \beta, \gamma, x, t)$ with another bifurcating phasetransition: from indifferent stability (dashed line) to bistability (solid line)

3. By initial assumption, the parameter $\alpha=\alpha(t)$ is time-varying, so it can be both positive $(\alpha(t) \geq 0$, with a single stable stationary state at the origin) and negative $(\alpha(t) \leq 0$, with two stable stationary states, symmetrically positioned left and right from the origin). Therefore, as the bifurcation parameter $\alpha$ changes from negative to positive values, our conflict system (6)-(7) evolves from a single stable stationary state $V(\alpha, \beta, x, t)_{\text {stable }}$ towards the double bistable stationary state $V(\alpha, \beta, x, t)_{\text {bistable }}-$ while passing through a sudden bifurcation change. This bistable phase is similar to Rubin's 'vase or face' Gestalt illusion. In the vicinity of this bifurcation phase-transition from stability to bistability of an individual conflict dynamics, we have a short chaotic interphase.

Therefore, each individual agent's behaviour, as described by the conflict model (6)-(7), evolves in its own conflict potential well $V(\alpha, \beta, x, t)$ according to the phase-transition map (see left panel in Figure 2):

Globally-Stable Goal-Directed Behaviour $\underset{\text { bifurcation }}{\longrightarrow}$ Bistable Conflicting Behaviour

Quintic forces. Finally, if we want to include some new psychological fields into an individual agent's conflicting behaviour, we can make a further perturbation expansion of the individual agent's kinematics (6)(7), by adding a third psychological field $\gamma(t) x(t)^{5}$, so that the velocity controller equation becomes:

$$
\dot{x}(t)=-\alpha(t) x(t)-\beta(t) x(t)^{3}-\gamma(t) x(t)^{5},
$$

with the corresponding conflict potential well:

$$
B=f(P, E, t) \Leftrightarrow B=V(\alpha, \beta, \gamma, x, t)=\frac{1}{2} \alpha(t) x(t)^{2}+\frac{1}{4} \beta(t) x(t)^{4}+\frac{1}{6} \gamma(t) x(t)^{6} .
$$

In this case, we have another form of bifurcating conflict potential well $V(\alpha, \beta, \gamma, x, t)$ (see right panel in Figure 2), depending on the new time-varying parameter $\gamma(t)$ :

Indifferently-Stable Goal-Directed Behaviour $\stackrel{\text { bifurcation }}{\longrightarrow}$ Bistable Conflicting Behaviour

Psychological interpretation of the conceptual model. The psychological interpretation of these phase transitions is the following: this represents a vacillation behaviour with a single source, rather than a colocated source and sink, which is required in the linear case, as illustrated in Figure 1 (right). The other effect of this bifurcation is the ability to dynamically transition from one behavioural regime to another, through the use of the control parameters $\alpha(t), \beta(t), \gamma(t)$, the agent's total mental state, $P=\left\{P_{a}\right\}=\{\alpha, \beta, \gamma\}$.

To illustrate the effect of this consider the attraction to some goal, say at the bottom right location of some landscape. Now consider a 'canyon' route with a cross-section as illustrated in Figure 2 (left) with $\alpha>0$ will result in a queuing behaviour of agents. If a threat is recognized colocated with the major goal then setting $\alpha<0$ will result in a vacillatory behaviour offset from the goal, which represents the psychological conflict of the indecision. The difference for the quintic indifferently stable goal case is that the 'canyon' is more like a 'promenade', meaning a group of agents will progress in a crowd milling action, rather than an orderly queue. 
Verification and validation of the conceptual model. The initial verification of Lewin's typology of psychological conflicts was conducted by Miller in the form of animal experimentation with laboratory rats (Miller, 1944). The qualitative analysis presented above shows the control parameter ranges for which Miller's results are reproduced and this model has been long regarded as a valid model of competition in decision making (Miller, 1944; Weiten, 1998).

The correct stand-off position in physical space where the agent's body must act is important. This must be decided on a case by case basis according to the context of the competition in decision making problem under consideration, and can either be assumptions of the model in those cases, see for instance (Millikan et al., 2013; Wong, 2012), or it might be based on experimental results. For example, recently Mezzacappa et al. have conducted laboratory experiments with students playing the role of violent rioting aggressors in groups attacking vehicles and other assets (Mezzacappa et al., 2011). It is in the detailed studies, like (Millikan et al., 2013; Wong, 2012), where the operations analyst must decide on the validity of the psychological model, i.e. as to whether or not psychological conflict in competition in decision making is an important factor contributing to the overall study.

\section{FUTURE WORK ON SOCIAL POWER-FIELDS}

In the previous section we have considered psychological conflict within the individual agent. Now we briefly turn to a question first posed by Helbing et al.(2000) which was how to model, physically, crowd turbulence (or, stampede), which appears to be the most dangerous collective behaviour of the panicking or rioting crowd. Helbing was exclusively concerned with crowd turbulence in physical space.

We can do little here now except to broadly outline a proposed plan for future work, which is to first show that noise-induced phase transitions, exist in this class of nonlinear DSC models, i.e., that the model is sufficiently rich that phase transitions can be represented at all; see (Johnson and Ivancevic, 2013). Essentially, this represents a model of social 'force-fields' or what Lewin called a power-field; i.e., those influences over individual behaviours that are induced by others as social-norms or social-dysfunctions and so on; see (Lewin, 1997, pp. 197-198).

\section{CONCLUSIONS}

Overall, there have been a number of aspects to the study. The particular conceptual-model we considered here was for competition in decision-making, where as a result psychological conflict might arise. In particular, we have applied previously formalized Lewinian force-field based socio-cognio-physics to a qualitative analysis of individual conflicting attractor behaviour within a general crowd, or artificial embodied agents, dynamics framework.

For this simple psychological conceptual model, the consequences of the field shape have been considered for a simple model of attraction and repulsion. We have been able to, in an executable computational model, reproduce the classic approach-avoid model of decision making in psychological conflict; see (Lewin, 1997; Weiten, 1998). Additionally we have been able to add a dynamic control parameter, $\alpha(t)$, to the traditionally static linear model (Weiten, 1998) of psychological forces, resulting in a spatio-temporal dynamic potential function $V(\alpha, x, t)$. Finally by adding some nonlinear parameters we have been able to produce a model which exhibits some quite rich nonlinear complex conflict behaviours and phase transitions. In the cubic case, now with control parameters $\alpha(t), \beta(t)$, the model is capable of producing bifurcating phase-transition from stability to bistability, while in the quintic case with control parameters $\alpha(t), \beta(t), \gamma(t)$, we can produce another bifurcating phase-transition, from indifferent stability to bistability.

For future work we have proposed the of modelling social-power-fields either using a ABM approach where social-groups are simple extensions of an individual agent or using a DSC approach where individual agents and/or whole social-groups are effectively swept around as 'turbulent' waves on some landscape.

\section{REFERENCES}

Balkenius, C. (1995). Natural Intelligence in Artificial Creatures. Lund, Sweden: Lund University Cognitive Studies, 37.

Castellano, C., S. Fortunato, and V. Loreto (2009). Statistical physics of social dynamics. Reviews of Modern Physics 2(81), 591-646. 
W.T. Johnson and V.G. Ivancevic, Individual Psychological Conflict within Social Group Dynamics

Conant, R. C. and W. R. Ashby (1970). Every good regulator of a system must be a model of that system. International journal of systems science 1(2), 89-97.

Epstein, S. (1978, October). Avoidance-approach: the fifth basic conflict. Journal of Consulting and Clinical Psychology 46(5), 1016-1022. PMID: 701540.

Ferber, J. (1999). Multi-Agent Systems: An Introduction to Distributed Artificial Intelligence. London: Addison-Wesley.

Helbing, D., I. Farkas, and T. Vicsek (2000, September). Simulating dynamical features of escape panic. Nature 407(6803), 487-490.

Ivancevic, V. G. and W. T. Johnson (2012). Macro-microscopic self-similarity in socio-cognio-physical dynamics. Journal | Mathematics in Engineering, Science and Aerospace 3(3), 325-339.

Ivancevic, V. G. and D. J. Reid (2010). Entropic geometry of crowd dynamics. In T. Evans (Ed.), Non-Linear Dynamics. Vienna: InTech.

Jager, W., R. Popping, and H. van de Sande (2001). Clustering and fighting in two- party crowds: Simulating the approach-avoidance conflict. Journal of Artificial Societies and Social Simulation 4(3), 7.

Johnson, W. T. and V. G. Ivancevic (2013). Progress toward a psycho-physical holistic embodied model of cognition. Technical Report (under review), DSTO, Edinburgh, Australia.

Johnson, W. T., M. Wong, and P. Jacques (2011, July). A study of issues involved in using an explicit cognitive model within an agent-based-model crowd scenario. In Invited Presentation Session on Holistic Approaches to Military and Security Modelling at IFORS 2011, Melbourne, Australia.

Lewin, K. (1935). Environmental forces in child behavior and development. In A Dynamic Theory of Personality: Selected Papers, McGraw-Hill Publications in Psychology, pp. 66-113. New York: McGraw-Hill Book Company.

Lewin, K. (1936). Principles of Topological Psychology. New York: McGraw-Hill Book Company.

Lewin, K. (1997). Resolving Social Conflicts and Field Theory in Social Science. Washington, DC.: American Psychological Association.

Mezzacappa, E., G. Cooke, G. Reid, R. DeMarco, C. Sheridan, and J. Riedener (2011). Mathematical capture of human crowd behavioral data for computational model building, verification, and validation. In Proceedings of the 20th Behavior Representation in Modeling and Simulation (BRIMS) Conference 2011, Sundance, Utah, pp. 215-222.

Miller, N. E. (1944). Experimental studies of conflict. In J. M. Hunt (Ed.), Personality and the Behavior Disorders: A handbook based on experimental and Clinical Research, Volume 1, pp. 431-465. New York: The Ronald Press Company.

Millikan, J., D. Grieger, and W. J. Chau (2011, July). From conceptual to holistic modelling: Societal responses of populations in crisis using an opinion dynamics model. In Invited Presentation Session on Holistic Approaches to Military and Security Modelling at IFORS 2011, Melbourne, Australia.

Millikan, J., M. Wong, and D. Grieger (2013). Suppression of dismounted soldiers: Towards improving dynamic threat assessment in closed loop combat simulations. In Proceedings of ModSim 2013, Adelaide, Australia.

Sun, R. (2008). The Cambridge handbook of computational psychology. Cambridge: Cambridge University Press.

Weiten, W. (1998). Psychology: Themes and Variations (4th ed.). Pacific Grove, CA.: Brooks/Cole Pub. Co.

Wong, M. (2012, June). Agent based crowd modelling for the usage of non-lethal weapons. In Proc. of SimTecT 2012, Adelaide, Australia. 